\title{
Teoría de juegos y la administración estratégica de empresas
}

\section{Theory of games and the strategic management of companies}

Said Diez Farhat

Universidad Católica de Santiago de Guayaquil, Ecuador

Lucia Pico Versoza

Universidad Internacional del Ecuador, Ecuador

Autor para correspondencia: said.diez@cu.ucsg.edu.ec, lupicove@uide.com.ec

Fecha de recepción: 04 de Agosto 2017 - Fecha de aceptación: 15 de Enero de 2018

Resumen

Esta investigación tiene como objetivo identificar las aplicaciones de la teoría de juegos en el proceso de dirección y administración estratégica de empresas. Las aplicaciones se enfocaron en las áreas de las finanzas, contabilidad y economía legal. Para el efecto se realizó una revisión de literatura de los conceptos, hallazgos y aportes de diversos autores que fueron recopilados de manera secuencial para ser analizados en el documento. Se concluye que la teoría de juegos tiene una participación activa en el proceso de dirección y administración estratégica de empresas para determinar los comportamientos irracionales o altruistas que pueden resultar productivos o no para la supervivencia de las organizaciones.

Palabras Clave: administración estratégica; teoría de juegos; estrategia; comportamiento racional; proceso de administración estratégica

\begin{abstract}
This research aims to identify applications of game theory in the process of strategic management and business administration. Applications focused on the areas of finance, accounting and legal economy. For this purpose a literature review of concepts, findings and contributions of various authors were sequentially collected to be analyzed in the document was made. It concludes that game theory has an active participation in the process of strategic management and business administration to determine irrational or altruistic behaviors that can be productive or not for the survival of organizations.
\end{abstract}

Key words: strategic management; game theory; strategy; rational behavior; strategic management process 


\section{Introducción}

Desde su nacimiento con Von Neumann y Morgenstern (1944), la Teoría de Juegos y Comportamiento Económico, y por las próximas dos décadas, se fundamentó especialmente en la disciplina de las matemáticas. Sin embargo, en los últimos 30 años se ha aplicado cada vez mayor en problemas prácticos, es decir a partir del análisis de competencia de la política monetaria; desde el diseño de las instituciones de subastas a la estructuración de incentivos dentro de las empresas; de las patentes como resolución de conflictos. En la actualidad la teoría de juegos es un elemento básico de los estudios de doctorados en economía, las finanzas y la ciencia de la administración.

A partir de lo anterior, se deduce que un requerimiento fundamental para la toma correcta de decisiones estratégicas de una empresa es evaluar las probables respuestas de los competidores a las alternativas de acción disponibles; además de considerar cómo la empresa espera que tales respuestas afecten los resultados de las acciones seleccionadas por ella. La teoría de los juegos estudia la toma racional de decisiones de los agentes cuando los resultados de las acciones seleccionadas por cada uno de ellos dependen, al menos en parte, de las acciones seleccionadas por otros. Es común, entonces, observar gran similitud entre los fenómenos estudiados en la teoría de los juegos y muchas decisiones estratégicas tomadas por las empresas (Tarziján \& Paredes, 2006, p. 184).

\section{Marco Teórico y Conceptual}

La teoría de juegos es el análisis del comportamiento racional en situaciones de interdependencia de los resultados, es decir que el pago de una persona depende de lo que haga la otra persona que se encuentra dentro del juego (Camerer, 1991). Su mayor impacto ha sido en economía, especialmente en los últimos 15 años en la organización industrial, pero su aplicación en otras disciplinas y áreas funcionales es demasiado activa. (Brandenburger \& Nalebuff, 1995). En cambio, la mayor parte de la investigación en la estrategia de los negocios es empírica, es decir, el enfoque tradicional es formar varias hipótesis basadas en los resultados de algunas teorías en la literatura. Posteriormente, las hipótesis se someten a pruebas de datos de corte transversal sobre las medidas de las firmas o unidades de negocio a nivel de rentabilidad, diversificación y publicidad (Behling \& Starke, 1973).

De acuerdo con Tarziján y Paredes (2006), se deduce que:

Un requerimiento fundamental para la toma correcta de decisiones estratégicas de una empresa es evaluar las probables respuestas de los competidores a las alternativas de acción disponibles; además de considerar cómo la empresa espera que tales respuestas afecten los resultados de las acciones seleccionadas por ella. La teoría de los juegos estudia la toma racional de decisiones de los agentes cuando los resultados de las acciones seleccionadas por cada uno de ellos dependen, al menos en parte, de las acciones seleccionadas por otros. Es común, entonces, observar gran similitud entre los fenómenos estudiados en la teoría de los juegos y muchas decisiones estratégicas tomadas por las empresas. (p. 184) 
La mayoría de los modelos se han centrado en cuestiones externas sobre las estrategias, recientemente estas herramientas han recurrido cada vez más a otras cuestiones con relación directa con la gestión estratégica que tiene que ver con la organización interna de la empresa y el alcance apropiado de sus actividades (Saloner, 1991). Estas herramientas incluyen preguntas relacionadas con el ámbito verticales de las actividades de la empresa (Grossman \& Hart, 1986); el efecto de los incentivos a la óptima el alcance horizontal de la empresa (Jensen 1986); y la amplitud apropiada de negocio de la empresa y las estrategias corporativas (Rotemberg \& Saloner, 1999b).

Para que un juego o situación estratégica esté definido completamente, deben establecerse los siguientes aspectos (Tarziján \& Paredes, 2006, p. 197):

- Jugadores.- Para que exista interacción estratégica, debe haber dos o más jugadores (empresas) que puedan interactuar.

- Reglas y estructura temporal del juego.- Deben definirse los movimientos (acciones) posibles a realizarse por cada jugador y su secuencialidad o simultaneidad. Además, deben especificarse aspectos tales como si el juego se juega sólo una vez o si se repite.

- Estructura de información del juego.- Debe especificarse lo que sabe cada jugador; por ejemplo, todos pueden tener la misma información o alguno de ellos poseer más información que el resto.

- Resultados del juego.- Deben conocerse los resultados que obtendrá cada uno de los jugadores por cada posible conjunto de acciones que realicen.

- Pagos a cada jugador.- Debe existir un pago predeterminado para cada uno de los jugadores en cada uno de los posibles resultados del juego.

Luego de entender los aspectos que deben establecerse para que un juego o situación estratégica este correctamente definido, se puede inferir que la teoría de los juegos ordena y formaliza los principios de la toma de decisiones estratégicas para la administración de una empresa que interactúa con sus competidores, empleados, consumidores y otros (Tarziján \& Paredes, 2006, p. 194). También se dice que no tiene como objetivo entregar respuestas a los ejecutivos respecto de cómo comportarse en cada situación estratégica ni reemplaza su intuición ni su experiencia, pero sí ayuda al ordenamiento y a la toma de decisiones estratégicas (Tarziján \& Paredes, 2006, p. 194).

El grado de racionalidad que se supone en los modelos de la teoría de juegos es a menudo mayor que en otros modelos económicos. En los modelos de la teoría de juegos la acción óptima de cada empresa depende de lo que se cree que sus rivales harían. Con el fin de decidir qué hacer en sí, la empresa debe ponerse en los zapatos de sus rivales y analizar la perspectiva de la situación de su rival. El análisis requiere, por tanto, suposiciones acerca de la racionalidad del rival, así como la evaluación de la creencia del rival. Estos supuestos son notables sobre todo en un campo como la gestión estratégica que tolera una amplia variedad de supuestos de comportamiento (Saloner, 1991).

Por otro lado, algunos autores explican que la teoría económica basada en la teoría de juegos sugiere: 
Que la depredación puede ser una estrategia posible y real cuando se den, de manera simultánea, las siguientes condiciones: (a) el potencial depredador opera simultáneamente en varios mercados, ya sea geográficos y/o de productos, (b) el potencial depredador baja sus precios después de que la entrada ha ocurrido en uno de los mercados en que haya entrada, y a estos precios la entrada se hace financieramente inviable para el entrante y (c) el eventual depredado no está seguro de que la baja en el precio del potencial depredador tenga como objetivo realizar depredación, y le asigna por lo menos una probabilidad positiva a que la baja en el precio sea producto de la competencia, o de menores costos (Tarziján \& Paredes, 2006, p. $360)$.

Una estrategia es un conjunto de metas y políticas importantes. La definición es tan simple como eso. Pero mientras la noción de una estrategia es extremadamente fácil de captar, la elaboración de un acuerdo sobre la declaración de una empresa determinada puede ser un aporte fundamental para el futuro éxito de la organización (Tilles, 1963). Con el fin de desarrollar una declaración, los administradores debe ser capaz de identificar con precisión lo que es entiende por una meta y lo que se entiende por una importante política (Ansoff, 1991). De lo contrario, el proceso de determinación de la estrategia puede degenerar en lo que tan a menudo se convierte en el registro solemne de lugares comunes, ya sea inútil para el esclarecimiento de la dirección o el logro de un consenso (Tilles, 1963).

Para el precursor de la administración estratégica, Ansoff (1965) estableció que "Las estrategias, son las expresiones operacionales de políticas en el sentido de que, dentro de un sistema administrativo, definen el criterio operacional sobre la base de cuales de los programas específicos pueden ser concebidos, seleccionados e implementados" (p. 43). Por lo tanto para entender el concepto de una estrategia, se debe conocer los siguientes criterios para que una estrategia funcione: (a) La consistencia interna, (b) Coherencia con el medio ambiente, (c) Adecuación de la disposición de recursos, (d) Grado satisfactorio de riesgo, (e) Adecuado horizonte temporal y (f) La trabajabilidad (Zayen, Hayar, \& Noubir, 2014). Sin embargo, la preocupación no son los aspectos cuantitativos pero con la identificación de algunos factores cualitativos que pueden servir como base para evaluar el grado de riesgo inherente a una estrategia. Estos factores son: (a) La cantidad de recursos (en la cual la estrategia se basa) cuya existencia continuada o valor no está asegurada, (b) La duración de los períodos de tiempo en que los recursos están comprometidos y (c) La proporción de los recursos comprometidos con un riesgo individual (Tilles, 1963).

De acuerdo con Tilles (1963), si una estrategia no puede ser evaluada por los resultados, hay algunos otros indicios de que puedan usarse para evaluar su contribución en el progreso de la empresa: (a) El grado de consenso que existe entre los ejecutivos de las metas y las políticas corporativas, (b) El grado en que las grandes áreas de gestión identifican con antelación, mientras que todavía hay tiempo para explorar una variedad de alternativas, (c) La medida en que las necesidades de recursos se descubren mucho antes del último minuto, lo que exige ni los programas de reducción de costos ni la eliminación de los programas previstos. Los enfoques de gran popularidad en la reducción de costes es una clara indicación del frecuente fracaso de la planificación estratégica corporativa. 
La administración estratégica como un campo de investigación es firmemente basada en la práctica y existe por la importancia de su sujeto. La administración estratégica de las organizaciones empresariales está en el corazón de la creación de la riqueza en la sociedad industrial moderna. La sociedad es servida por las organizaciones eficientes y la administración estratégica se refiere a la entrega a través del estudio de su creación, el éxito y supervivencia, así como con la comprensión de su fracaso, sus costos, y su lecciones (Rumelt, Schendel, \& Teece, 1991). El proceso de la administración estratégica se compone de los siguientes pasos (David, 2011; Hahn, 2013; Wheelen \& Hunger, 2012):

- Explorar el ambiente.- Realizar auditoria interna y externa

- Formulación de la estrategia.- Desarrollar la misión y visión, Establecer los objetivos a largo plazo y Generar las estrategias

- Implementación de la estrategia

- Evaluación de la estrategia.- Medir y evaluar el desempeño

\section{Materiales y Métodos}

Para establecer las aplicaciones de la teoría de juegos en la administración estratégica se efectuó una recopilación de las explicaciones y aportes conceptuales de diversos autores considerados los gurús en el campo de la administración y las estrategias. Los materiales utilizados fueron artículos científicos publicados en los mejores journals de administración de empresas y negocios, así como libros de los precursores del tema analizado, a fin de obtener dicha información, se consultó la base de datos de Thomson Reuters y Scopus mediante la búsqueda de las palabras claves como Strategic Management, Game Theory, Strategy, Rational Behavior y Strategic Management Process.

\section{Análisis y Discusión}

La incapacidad de las teorías estándar en las finanzas para proporcionar una explicación satisfactoria para fenómenos observados condujo a una búsqueda de teorías que utilizan las nuevas metodologías. Esta fue particularmente cierto en las finanzas corporativas, donde los modelos existentes eran tan claramente insatisfactorio. La teoría de juegos ha proporcionado una metodología que ha traído ideas en muchos fenómenos inexplicables con anterioridad al permitir que la información asimétrica y la interacción estratégica para ser incorporado en el análisis. Se discute sobre el uso de la teoría de juegos en finanzas corporativas donde hasta la fecha ha sido aplicada con mayor éxito (Chatterjee \& Samuelson, 2014).

Sería vano pretender que el enfoque de la teoría de juegos en la contabilidad tiene respuestas definitivas que se pueden implementar en un sentido práctico. Aunque se han obtenido importantes conocimientos, existe mucho trabajo adicional que hay que hacer antes de que la literatura pueda traducirse en recomendaciones. Para ilustrar el efecto de la teoría de juegos en la contabilidad, Chatterjee y Samuelson (2014) expresaron lo siguiente:

Supongamos que una empresa de venta al por menor contrata a 20 vendedores, con trabajo aparentemente similar calificaciones, para vender productos similares en ambientes similares. En teoría, la empresa debe ofrecer diferentes contratos a cada vendedor a pesar que 
todos se dedican al mismo trabajo. Las realidades políticas y administrativas hacen esta recomendación difícil de implementar. Una segunda, y tal vez más grave, limitación de la teoría es la suposición de que el director conoce las preferencias perfectamente de cada agente y que el contrato se pueda cambiar cada vez que el agente cambia sus preferencias. Siendo realistas, las preferencias de un individuo son tan privadas y tan volátiles que incluso no revela plenamente las preferencias. Estas son limitaciones serias; sin embargo, la teoría proporciona valiosa ideas sobre cuestiones prácticas que ninguna empresa puede escapar. La teoría establece que el costo de confiar en las medidas de los rendimientos surge de la imposición de riesgo en el agente, y no de una falta de control. (p. 76)

Existe una amplia bibliografía que considera el desarrollo acotado de los consumidores de la racionalidad como una forma de costos de búsqueda, y examina sus implicaciones para cosas tales como la opacidad del precio. El efecto de la opacidad de los precios en los consumidores ha sido conocida desde hace tiempo: los consumidores cometen errores cuando tienen que decodificar los precios presentados en formatos complicados, y las empresas pueden utilizar esto para aumentar los ingresos (Ellison 2005; Ellison \& Ellison 2009). Se ha revisado algunas aplicaciones de la teoría de juegos en la comercialización, centrándose en las ideas claves que la teoría de juegos ha traído a la estrategia de marketing. Los estudios empíricos en el marketing son cada vez más de carácter estructural, y llevan a la oferta y a la demanda a estar relacionados (Chatterjee \& Samuelson, 2014).

Se han desarrollado los modelos de la teoría de juegos en el ámbito de la negociación que sufren una serie de deficiencias teóricas y supuestos fuertes. El objetivo de los investigadores debe ser para remediar estas deficiencias con mejores modelos (Brandenburger \& Nalebuff, 1995). Sin embargo, la teoría sigue siendo valiosa, no porque proporciona predicciones cuantitativas agudas de los resultados en las negociaciones reales, sino porque nos da información cualitativa sobre el comportamiento (Blumentritt, Mathews, \& Marchisio, 2013).

Por su propia naturaleza, la teoría es contingente y señala que las diferencias aparentemente menores, incluso en una situación de negociación dado pueden cambiar dramáticamente la naturaleza del juego que se está reproduciendo. Y la teoría también sugiere la dirección de dicho cambio y lo que se podría hacer al respecto. No hay nada que pueda sustituir a un estudio cuidadoso y preparación para una negociación. El conocimiento de la teoría proporciona categorías para clasificar el material que se tiene sobre una situación y para analizar el contenido (Chatterjee \& Samuelson, 2014).

Las críticas sugieren que las leyes y la economía no están exentas de costos. El equilibrio entre la necesidad del realismo. Es importante identificar si seguir evaluando los escenarios legales puede ser analizado en términos de la teoría de juegos (Tian, Govindan, \& Zhu, 2014). Sin embargo, el reconocimiento de algunas deficiencias en la ley y la economía no justifica la idea de ignorarlo. Cualquiera que sea sus costos, leyes y economía también tienen algunas ventajas, la teoría de juegos puede contribuir de forma importante a la comprensión de los efectos de las leyes, de cómo esas leyes podrían servir para mejora el objetivo de la eficiencia (Saghezchi, Saghezchi, Nascimento, \& Rodriguez, 2014). 
Finalmente, luego de haber analizado el desarrollo de los modelos de la teoría de juegos en los distintos ámbitos académicos y prácticos, la teoría de juegos es la mejor opción para resolver las implicaciones lógicas de un agente en el pensamiento racional extendido sobre lo que el otro va a hacer, como parte de su proceso de decisión (Aplak \& Sogut, 2013). Esas consecuencias lógicas sin duda deben ser de interés para las estrategias de los investigadores, ya que muchos aspectos de la formulación de la estrategia deben ajustarse al modelo de la teoría de juegos. Pero no son los únicos procesos de pensamiento estratégico de interés (Camerer, 1991).

\section{Conclusiones}

Luego de la presentación de una exhaustiva revisión de literatura, se puede concluir que la teoría de juegos tiene una participación activa en el proceso de dirección y administración estratégica de empresas, debido que con este aprendizaje se puede ayudar a explicar porque ciertos comportamientos irracionales o altruistas resultan ser productivos y porque las empresas que se dedican a estos comportamientos sobreviven y prosperan en un mundo tan competitivo.

\section{Bibliografía}

Ansoff, I. (1965). The Corporate Strategy. New York, USA: Mc Graw Hill.

Ansoff, I. (1991). Critique of Henry Mintzberg's "The design school: Reconsidering the basic premises of strategic management." Strategic Management Journal, 12(6), 449-461. http://doi.org/10.1002/smj.4250120605

Aplak, H., \& Sogut, M. (2013). Game theory approach in decisional process of energy management for industrial sector. Energy Conversion and Management, 74(3), 70-80. http://doi.org/10.1016/j.enconman.2013.03.027

Blumentritt, T., Mathews, T., \& Marchisio, G. (2013). Game Theory and Family Business Succession An Introduction. Family Business Review, 26(1), 51-67.

http://doi.org/10.1177/0894486512447811

Brandenburger, A., \& Nalebuff, B. (1995). The Right Game - Use Game-Theory to Shape Strategy. Harvard Business Review, 73(4), 57-71.

Camerer, C. (1991). Does Strategy Research Need Game Theory? Strategic Management Journal, 12(1), 137-152.

Chatterjee, K., \& Samuelson, W. (Eds.). (2014). Game theory and business applications (2. ed). Berlin: Springer.

David, F. (2011). Strategic management: Concepts and cases, 13th edition. Prentice Hall: Upper Saddle River, N.J.

Ellison, G. (2005). A model of add-on pricing. Quarterly Journal of Economics, 120(1), 585-637. 
Ellison, G., \& Ellison, S. (2009). Search, obfuscation, and price elasticities on the internet. Econometrical, 77(2), 427-452.

Grossman, S. \& Hart, O. (1986). The costs and benefits of ownership: A theory of vertical and lateral integration". Journal of Political Economy, 94(1), 691-719.

Hahn, R. (2013). ISO 26000 and the Standardization of Strategic Management Processes for Sustainability and Corporate Social Responsibility. Business Strategy and the Environment, 22(7), 442-455. http://doi.org/10.1002/bse.1751

Jensen, M. (1986). Agency costs of free cash flow, corporate finance and takeovers", American Economic Review, 76(1), 323-329.

Rotemberg, J. \& Saloner, G. (1986). A Supergame theoretic model of price-wars during bottoms. American Economic Review, 76(1), 390-407.

Rumelt, R., Schendel, D., \& Teece, D. (1991). Strategic Management and Economics. Strategic Management Journal, 12(1), 5-29.

Saghezchi, F., Saghezchi, F., Nascimento, A., \& Rodriguez, J. (2014). Game Theory and Pricing Strategies for Demand-Side Management in the Smart Grid. 2014 9th International Symposium on Communication Systems, Networks \& Digital Signal Processing (Csndsp), 883-887.

Saloner, G. (1991). Modeling, game theory, and strategic management. Strategic Management Journal, 12(2), 119-136. http://doi.org/10.1002/smj.4250121009

Tarziján, J., \& Paredes, R. (2006). Organización industrial para la estrategia empresarial (2a ed.). México: Pearson Education.

Tian, Y., Govindan, K., \& Zhu, Q. (2014). A system dynamics model based on evolutionary game theory for green supply chain management diffusion among Chinese manufacturers. Journal of Cleaner Production, 80(1), 96-105. http://doi.org/10.1016/j.jclepro.2014.05.076

Tilles, S. (1963). How to Evaluate Corporate Strategy. Harvard Business Review, 41(4), 111-121.

Von Neumann, J., \& Morgenstern, O. (1944). Theory of games and economic behavior (First edition). Princeton, N.J.; Woodstock: Princeton University Press.

Wheelen, T., Hunger, J. (2012). Strategic management and business policy: Toward global sustainability (13th edition). Pearson Prentice Hall: Upper Saddle River, N.J.

Zayen, B., Hayar, A., \& Noubir, G. (2014). Game theory-based resource management strategy for cognitive radio networks. Multimedia Tools and Applications, 70(3), 2063-2083. http://doi.org/10.1007/s11042-012-1211-0 